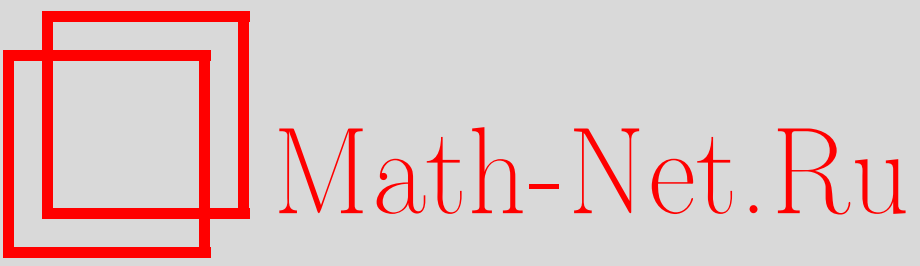

Е. А. Марушкина, Е. С. Самсонова, Локальная динамика пары уравнений Хатчинсона с конкурентной и диффузионной связью, Итоги науки и техн. Сер. Соврем. мат. и ее прил. Темат. обз., 2021, том 194, 155-162

DOI: https://doi.org/10.36535/0233-6723-2021-194-155-162

Использование Общероссийского математического портала Math-Net.Ru подразумевает, что вы прочитали и согласны с пользовательским соглашением

http://www.mathnet.ru/rus/agreement

Параметры загрузки:

IP: 52.23 .180 .231

26 апреля 2023 г., 10:23:23 


\title{
ЛОКАЛЬНАЯ ДИНАМИКА ПАРЫ УРАВНЕНИЙ ХАТЧИНСОНА С КОНКУРЕНТНОЙ И ДИФФУЗИОННОЙ СВЯЗЬЮ
}

\author{
(c) 2021 г. $\quad$ E. А. МАРУШКИНА, Е. С. САМСОНОВА
}

\begin{abstract}
АннотАция. Изучается динамика системы, состоящей из двух связанных уравнений Хатчинсона с учетом конкурентной и диффузионной связи между популяциями. Выполнен локальный асимптотический анализ системы в случае малости коэффициентов связи и близости параметров осцилляторов к значениям, при которых происходит бифуркация Андронова-Хопфа. В работе анализируется сценарий фазовых перестроек системы при изменении параметра диффузии и исследуется зависимость этого сценария от коэффициента конкурентной связи.
\end{abstract}

Ключевые слова: уравнение Хатчинсона, конкурентная связь, диффузионная связь, метод нормальных форм, асимптотика, устойчивость.

\section{LOCAL DYNAMICS OF A PAIR OF HUTCHINSON EQUATIONS WITH COMPETITIVE AND DIFFUSION COUPLING}

\author{
(c) 2021 E. A. MARUSHKINA, E. S. SAMSONOVA
}

\begin{abstract}
In this paper, we study the dynamics of a system consisting of two coupled Hutchinson equations taking into account the competitive and diffusion coupling between populations. A local asymptotic analysis of the system is performed in the case where the coupling coefficients are small and the parameters of the oscillators are close to the values that provide the Andronov-Hopf bifurcation. We also examine the scenario of phase rearrangements of the system under a change in the diffusion parameter and analyze the dependence of this scenario on the coefficient of competition coupling.
\end{abstract}

Keywords and phrases: Hutchinson equation, competitive coupling, diffusion coupling, method of normal forms, asymptotics, stability.

AMS Subject Classification: 37G05, 37G10, 37G15, 34K20

1. Постановка задачи. Рассмотрим систему связанных уравнений Хатчинсона:

$$
\left\{\begin{array}{l}
\dot{N}_{1}=r\left[1+\alpha-\frac{N_{1}(t-h)}{k}-\alpha \frac{N_{2}}{k}\right] N_{1}+d\left(N_{2}-N_{1}\right), \\
\dot{N}_{2}=r\left[1+\alpha-\frac{N_{2}(t-h)}{k}-\alpha \frac{N_{1}}{k}\right] N_{2}+d\left(N_{1}-N_{2}\right) .
\end{array}\right.
$$

Здесь $N_{1}(t), N_{2}(t)$ - плотности численностей популяций, $r$-мальтузианский коэффициент линейного роста, $h$ - возраст половозрелости особей, $k$ - средняя плотность популяции. Параметр связи $\alpha$ отвечает за конкуренцию видов, а $d$ - коэффициент диффузионного взаимодействия.

Уравнение Хатчинсона, впервые предложенное в [9], является простейшим способом учета возрастной структуры в задаче о динамике популяции особей, борющихся за общую пищу. Система (1) при $\alpha=0$ подробно изучена в $[1,3,8]$. В [5] рассмотрена модель слабого конкурентного

Работа выполнена при поддержке Российского фонда фундаментальных исследований (проект № 18-29-10043). 
взаимодействия близких видов. Отметим, что при значении $r h$, близком к $\pi / 2$, и $\alpha=d=0$ у каждого из уравнений системы (1) от единичного состояния равновесия ответвляется орбитально устойчивый цикл (см. $[2,6,7])$. Наша цель - изучить систему (1) при значениях параметров $r$ и $h$, близких к критическим, и достаточно малых $\alpha$ и $d$.

Замена $N_{j}=k\left(1+x_{j}\right), j=1,2$, и переобозначения $t h \rightarrow t$ и $r h \rightarrow r$ приводят к системе

$$
\left\{\begin{array}{l}
\dot{x}_{1}=-r\left(1+x_{1}\right)\left(x_{1}(t-1)+\alpha x_{2}\right)+d\left(x_{2}-x_{1}\right), \\
\dot{x}_{2}=-r\left(1+x_{2}\right)\left(x_{2}(t-1)+\alpha x_{1}\right)+d\left(x_{1}-x_{2}\right),
\end{array}\right.
$$

нулевое решение которой соответствует решению $N_{1}=N_{2}=k$ системы (1).

Предположим, что параметр $r$ близок к критическому значению, $r=\pi / 2+\varepsilon$, а коэффициенты связи $\alpha>0$ и $d>0$ пропорциональны величине надкритичности $0<\varepsilon \ll 1$. Таким образом, рассмотрим систему

$$
\left\{\begin{array}{l}
\dot{x}_{1}=-\left(\frac{\pi}{2}+\varepsilon\right)\left(x_{1}(t-1)+\varepsilon \alpha x_{2}\right)\left(1+x_{1}\right)+\varepsilon d\left(x_{2}-x_{1}\right), \\
\dot{x}_{2}=-\left(\frac{\pi}{2}+\varepsilon\right)\left(x_{2}(t-1)+\varepsilon \alpha x_{1}\right)\left(1+x_{2}\right)+\varepsilon d\left(x_{1}-x_{2}\right) .
\end{array}\right.
$$

Задача об устойчивости нулевого решения системы (3) приводит к характеристическому уравнению вида

$$
\left(\lambda+\left(\frac{\pi}{2}+\varepsilon\right) e^{-\lambda}+2 \varepsilon d-\left(\frac{\pi}{2}+\varepsilon\right) \varepsilon \alpha\right)\left(\lambda+\left(\frac{\pi}{2}+\varepsilon\right) e^{-\lambda}+\left(\frac{\pi}{2}+\varepsilon\right) \varepsilon \alpha\right)=0,
$$

для которого выполнено следующее утверждение.

Лемма 1. Для всех достаточно малых в характеристическое уравнение (4) имеет две пары собственных чисел, близких к мнимой оси, с асимптотикой

$$
\begin{aligned}
& \lambda_{1,2}= \pm i \frac{\pi}{2}+\frac{\varepsilon}{1+\frac{\pi^{2}}{4}}\left[\frac{\pi}{2}(\alpha+1)-2 d \pm i\left(1-\frac{\pi^{2}}{4} \alpha+\pi d\right)\right]+O\left(\varepsilon^{2}\right), \\
& \lambda_{3,4}= \pm i \frac{\pi}{2}+\frac{\varepsilon}{1+\frac{\pi^{2}}{4}}\left[\frac{\pi}{2}(1-\alpha) \pm i\left(1+\frac{\pi^{2}}{4} \alpha\right)\right]+O\left(\varepsilon^{2}\right) .
\end{aligned}
$$

Все остальные корни этого уравнения лежат в левой комплексной полуплоскости.

Асимптотика корней характеристического уравнения (5)-(6) позволяет утверждать, что устойчивость нулевого решения системы (3) при достаточно малом $\varepsilon$ зависит от знаков величин $\pi(\alpha+1) / 2-2 d$ и $\pi(1-\alpha) / 2$. При $\varepsilon>0$ положительность любого из этих чисел влечет неустойчивость нулевого решения, а их отрицательность - устойчивость.

2. Построение нормальной формы. При $\varepsilon=0$ система (3) имеет устойчивое четырехмерное инвариантное многообразие, уравнения на котором могут быть получены с помощью метода нормальных форм. Для нахождения нормальной формы задачи выполним стандартную замену (cm. $[3,4])$

$$
x_{j}(t)=\sqrt{\varepsilon}\left(z_{j}(\tau) e^{i \frac{\pi}{2} t}+\text { к.c. }\right)+\varepsilon u_{j 1}(t, \tau)+\varepsilon^{3 / 2} u_{j 2}(t, \tau)+\ldots,
$$

где $z_{j}(\tau)$ - комплекснозначные функции медленного времени $\tau=\varepsilon t(j=1,2)$. Последовательно приравнивая коэффициенты при одинаковых степенях $\sqrt{\varepsilon}$ и учитывая разложение

$$
z_{j}(\tau-\varepsilon)=z_{j}(\tau)-\varepsilon z_{j}^{\prime}(\tau)+\ldots,
$$

получаем при $\sqrt{\varepsilon}$ верное тождество.

Отметим, что при $d=\alpha=0$ для каждого из осцилляторов $x_{1}(t), x_{2}(t)$ выполнено следующее утверждение (см., например, $[1,3,8]$ ).

Теорема 1. Существует такое $\varepsilon_{0}>0$, что для всех $0<\varepsilon \leqslant \varepsilon_{0}$ уравнение

$$
\dot{x}=-\left(\frac{\pi}{2}+\varepsilon\right) x(t-1)(1+x)
$$


имеет в окрестности нулевого состояния равновесия орбитально асимптотически устойчивый иикл с асимптотикой

$$
x(t, \varepsilon)=\sqrt{\varepsilon}\left(\sqrt{\frac{10}{3 \pi-2}} \exp \left(i\left(\frac{\pi}{2}+\varepsilon \frac{2}{3 \pi-2}\right) t\right)+\kappa . c .\right)+\varepsilon u(t, \tau)+O\left(\varepsilon^{3 / 2}\right),
$$

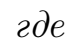

$$
u=\frac{2-i}{5} z^{2} e^{i \pi t}+\frac{2+i}{5} \bar{z}^{2} e^{-i \pi t}, \quad z=\sqrt{\frac{10}{3 \pi-2}} e^{i \varepsilon \frac{2}{3 \pi-2} t} .
$$

На втором шаге алгоритма при $\varepsilon$ возникает система уравнений на $u_{j 1}(t, \tau)$, идентичная соответствующей системе для одного осциллятора:

$$
\left\{\begin{array}{l}
\dot{u}_{11}=-\frac{\pi}{2} u_{11}(t-1)+\frac{\pi}{2}\left(z_{1}^{2} e^{i \pi t}+\bar{z}_{1}^{2} e^{-i \pi t}\right) \\
\dot{u}_{21}=-\frac{\pi}{2} u_{21}(t-1)+\frac{\pi}{2}\left(z_{2}^{2} e^{i \pi t}+\bar{z}_{2}^{2} e^{-i \pi t}\right)
\end{array}\right.
$$

решение которой имеет вид

$$
\begin{aligned}
& u_{11}=\frac{2-i}{5} z_{1}^{2} e^{i \pi t}+\frac{2+i}{5} \bar{z}_{1}^{2} e^{-i \pi t}, \\
& u_{21}=\frac{2-i}{5} z_{2}^{2} e^{i \pi t}+\frac{2+i}{5} \bar{z}_{2}^{2} e^{-i \pi t} .
\end{aligned}
$$

На третьем шаге алгоритма из условий разрешимости задач для $u_{j 2}(t, \tau)$ в классе 4 -периодических по $t$ функций получим следующую нормальную форму:

$$
\left\{\begin{array}{l}
\left(1+i \frac{\pi}{2}\right) z_{1}^{\prime}=i z_{1}+\frac{(1-3 i) \pi}{10} z_{1}\left|z_{1}\right|^{2}-\frac{\pi}{2} \alpha z_{2}+d\left(z_{2}-z_{1}\right), \\
\left(1+i \frac{\pi}{2}\right) z_{2}^{\prime}=i z_{2}+\frac{(1-3 i) \pi}{10} z_{2}\left|z_{2}\right|^{2}-\frac{\pi}{2} \alpha z_{1}+d\left(z_{1}-z_{2}\right),
\end{array}\right.
$$

грубым решениям которой соответствуют грубые решения системы (3) той же устойчивости с асимптотикой (7).

3. Анализ нормальной формы. Перейдем в системе (10) к полярным переменным, выполнив для этого замену

$$
z_{j}=\sqrt{\frac{10|1-\alpha|}{3 \pi-2}} \xi_{j} e^{i \varphi_{j}}, \quad j=1,2 .
$$

После подстановки (11) получим следующую систему уравнений:

$$
\begin{aligned}
\left(1+\frac{\pi^{2}}{4}\right)\left(\xi_{1}^{\prime}+i \varphi_{1}^{\prime} \xi_{1}\right) & =\left(\frac{\pi}{2}(1-\alpha)+i\left(1+\alpha \frac{\pi^{2}}{4}\right)\right) \xi_{1}+\frac{\pi|1-\alpha|\left(1-\frac{3 \pi}{2}-i\left(\frac{\pi}{2}+3\right)\right)}{3 \pi-2} \xi_{1}^{3}+ \\
& +\left(d-\frac{\pi}{2} \alpha\right)\left(1-i \frac{\pi}{2}\right)\left(\xi_{2}\left(\cos \left(\varphi_{2}-\varphi_{1}\right)+i \sin \left(\varphi_{2}-\varphi_{1}\right)\right)-\xi_{1}\right), \\
\left(1+\frac{\pi^{2}}{4}\right)\left(\xi_{2}^{\prime}+i \varphi_{2}^{\prime} \xi_{2}\right) & =\left(\frac{\pi}{2}(1-\alpha)+i\left(1+\alpha \frac{\pi^{2}}{4}\right)\right) \xi_{2}+\frac{\pi|1-\alpha|\left(1-\frac{3 \pi}{2}-i\left(\frac{\pi}{2}+3\right)\right)}{3 \pi-2} \xi_{2}^{3}+ \\
& +\left(d-\frac{\pi}{2} \alpha\right)\left(1-i \frac{\pi}{2}\right)\left(\xi_{1}\left(\cos \left(\varphi_{2}-\varphi_{1}\right)-i \sin \left(\varphi_{2}-\varphi_{1}\right)\right)-\xi_{2}\right) .
\end{aligned}
$$

Введем обозначение $\varphi=\varphi_{2}-\varphi_{1}$; приравнивая действительные и мнимые части уравнений, получим следующую систему:

$$
\begin{aligned}
& \left(1+\frac{\pi^{2}}{4}\right) \xi_{1}^{\prime}=\frac{\pi}{2}(1-\alpha) \xi_{1}-\frac{\pi}{2}|1-\alpha| \xi_{1}^{3}+\left(d-\frac{\pi}{2} \alpha\right)\left(\xi_{2}\left(\cos \varphi+\frac{\pi}{2} \sin \varphi\right)-\xi_{1}\right) \\
& \left(1+\frac{\pi^{2}}{4}\right) \xi_{2}^{\prime}=\frac{\pi}{2}(1-\alpha) \xi_{2}-\frac{\pi}{2}|1-\alpha| \xi_{2}^{3}+\left(d-\frac{\pi}{2} \alpha\right)\left(\xi_{1}\left(\cos \varphi-\frac{\pi}{2} \sin \varphi\right)-\xi_{2}\right) \\
& \left(1+\frac{\pi^{2}}{4}\right) \varphi^{\prime}=-\frac{\pi(\pi+6)|1-\alpha|}{2(3 \pi-2)}\left(\xi_{2}^{2}-\xi_{1}^{2}\right)-\left(d-\frac{\pi}{2} \alpha\right)\left(\frac{\xi_{1}}{\xi_{2}}\left(\sin \varphi+\frac{\pi}{2} \cos \varphi\right)+\frac{\xi_{2}}{\xi_{1}}\left(\sin \varphi-\frac{\pi}{2} \cos \varphi\right)\right)
\end{aligned}
$$


Заметим, что

$$
\cos \varphi \pm \frac{\pi}{2} \sin \varphi=\sqrt{1+\pi^{2} / 4} \cos (\varphi \pm \delta), \quad \sin \varphi \mp \frac{\pi}{2} \cos \varphi=\sqrt{1+\pi^{2} / 4} \sin (\varphi \pm \delta),
$$

где $\delta=-\operatorname{arctg}(\pi / 2)$. Тогда, выполнив замену

$$
\tau=\frac{2 \pi|1-\alpha|}{\pi^{2}+4} s
$$

получим нормальную форму, записанную в амплитудных и фазовых переменных:

$$
\begin{aligned}
\xi_{1}^{\prime} & =\operatorname{sgn}(1-\alpha) \xi_{1}-\xi_{1}^{3}+D\left(\xi_{2} \cos (\varphi+\delta)-\xi_{1} \cos \delta\right), \\
\xi_{2}^{\prime} & =\operatorname{sgn}(1-\alpha) \xi_{2}-\xi_{2}^{3}+D\left(\xi_{1} \cos (\varphi-\delta)-\xi_{2} \cos \delta\right), \\
\varphi^{\prime} & =-b\left(\xi_{2}^{2}-\xi_{1}^{2}\right)-D\left(\frac{\xi_{1}}{\xi_{2}} \sin (\varphi-\delta)+\frac{\xi_{2}}{\xi_{1}} \sin (\varphi+\delta)\right),
\end{aligned}
$$

где

$$
b=\frac{\pi+6}{3 \pi-2}, \quad D=\sqrt{1+\frac{\pi^{2}}{4}} \frac{2 d-\pi \alpha}{\pi|1-\alpha|} .
$$

Для нормальной формы (12) имеет место стандартное утверждение (см., например, [4]) о соответствии ее грубых режимов решениям исходной системы (3).

Теорема 2. Пусть $\left(\xi_{1}(s), \xi_{2}(s), \varphi(s)\right)$ - асимптотически устойчивое состояние равновесия (иикл) системы (12). Тогда существует такое $\varepsilon_{0}>0$, что для любого $0<\varepsilon \leqslant \varepsilon_{0}$ система (3) имеет экспоненциально орбитально устойчивый цикл (двумерный тор) с асимптотикой, определяемой заменами (7) и (11).

Система (12) получена в максимально простом виде. Функции $\xi_{1}(s)$ и $\xi_{2}(s)$ представляют собой медленно меняющиеся амплитуды циклов, а $\varphi(s)$ - их разность фаз. Отметим, что фазовое пространство системы (12) является цилиндрическим в силу $2 \pi$-периодичности правых частей по $\varphi$. Кроме того, имеется симметрия фазового пространства, состоящая в том, что замена

$$
\xi_{1} \rightarrow \xi_{2}, \quad \xi_{2} \rightarrow \xi_{1}, \quad \varphi \rightarrow-\varphi
$$

переводит исследуемую систему в себя. Более того, система (12) инвариантна относительно преобразования

$$
\xi_{1} \rightarrow \xi_{2}, \quad \xi_{2} \rightarrow \xi_{1}, \quad \delta \rightarrow-\delta, \quad b \rightarrow-b .
$$

4. Динамика нормальной формы. При анализе динамических свойств системы (12) удобно выделить два случая: $0<\alpha<1$ и $\alpha>1$. Если $0<\alpha<1$, то нормальная форма (12) может быть переписана в виде

где

$$
\begin{aligned}
\xi_{1}^{\prime} & =\xi_{1}-\xi_{1}^{3}+D\left(\xi_{2} \cos (\varphi+\delta)-\xi_{1} \cos (\delta)\right) \\
\xi_{2}^{\prime} & =\xi_{2}-\xi_{2}^{3}+D\left(\xi_{1} \cos (\varphi-\delta)-\xi_{2} \cos (\delta)\right) \\
\varphi^{\prime} & =-b\left(\xi_{2}^{2}-\xi_{1}^{2}\right)-D\left(\frac{\xi_{1}}{\xi_{2}} \sin (\varphi-\delta)+\frac{\xi_{2}}{\xi_{1}} \sin (\varphi+\delta)\right)
\end{aligned}
$$

$$
D=\frac{2 d-\pi \alpha}{\pi(1-\alpha)} \sqrt{1+\frac{\pi^{2}}{4}} .
$$

Рассмотрим случай, когда $\xi_{1}=\xi_{2}$. Тогда система (13) имеет два состояния равновесия $\xi_{1}=\xi_{2}=1, \varphi=0$ и $\xi_{1}=\xi_{2}=\xi^{*}=\sqrt{1-2 D \cos \delta}, \varphi=\pi$. Кроме того, в этой ситуации данная система полностью соответствует системе, полученной в работе [1] в качестве нормальной формы системы диффузионно слабо связанных уравнений Хатчинсона. Отметим, что в [1] приведен анализ ее качественного поведения при значениях $D>0$ и выделены области с разными сценариями фазовых перестроек при изменении параметра $D$.

Чтобы разобраться с фазовыми перестройками, рассмотрим вопрос о бифуркациях, происходящих с состояниями равновесия $\xi_{1}=\xi_{2}=1, \varphi=0$ и $\xi_{1}=\xi_{2}=\xi^{*}=\sqrt{1-2 D \cos \delta}, \varphi=\pi$. 
Следующее утверждение (см. [1]) позволяет выяснить, при каких условиях от состояния равновесия $\xi_{1}=\xi_{2}=1, \alpha=0$ ответвляется пара состояний равновесия.

Теорема 3. Пусть $D-D_{\kappa p}<0$, әде

$$
\begin{gathered}
c \equiv \frac{1}{D_{\kappa p}}\left(2 b \sqrt{1-\cos ^{2} \delta}\left(b^{2} \cos \delta-5 \cos \delta+2\right)+2\left(3 \cos ^{2} \delta-1\right)\left(b^{2}+1\right)\right), \\
D_{\kappa p}=-\cos \delta-b \sin \delta,
\end{gathered}
$$

тогда в достаточно малой окрестности неподвижной точки $(1,1,0)^{T}$ имеются два устойчивых состояния равновесия $\left(\xi_{1}^{*}, \xi_{2}^{*}, \alpha^{*}\right)^{T}$ u $\left(\xi_{2}^{*}, \xi_{1}^{*},-\alpha^{*}\right)^{T}$. Величины $\xi_{1}^{*}, \xi_{2}^{*}$, $\alpha^{*}$ допускают при $\left|D-D_{к р}\right| \ll 1$ асимптотическое представление

$$
\begin{aligned}
\xi_{1}^{*} & =1+\sqrt{\left(D-D_{\kappa p}\right) / c}+O\left(D-D_{\kappa p}\right), \\
\xi_{2}^{*} & =1-\sqrt{\left(D-D_{\kappa p}\right) / c}+O\left(D-D_{\kappa p}\right), \\
\alpha^{*} & =-2(1+D \cos \delta) \sqrt{\frac{D-D_{\kappa p}}{c\left(1-\cos ^{2} \delta\right)}}+O\left(D-D_{\kappa p}\right) .
\end{aligned}
$$

Условия устойчивости состояния равновесия $\xi_{1}=\xi_{2}=\sqrt{1-2 D \cos \delta}, \alpha=\pi$ позволяют определить значение $D_{\pi}=1 /(4 \cos \delta)$ такое, что при $D>D_{\pi}$ данная неподвижная точка неустойчива, а при $D<D_{\pi}$ - устойчива.

Положим $\sigma=D-D_{\pi}$; рассмотрим характер потери устойчивости данным состоянием равновесия. В частности, выполнена следующая теорема (см. [1]).

Теорема 4. Пусть $\sigma d_{\pi, 0}<0$, где

$$
d_{\pi, 0}=-\frac{3\left(-3+3 b^{2}+\left(1+3 b^{2}\right) \cos 2 \delta+2 b \sin 2 \delta\right)}{8(-2 b \cos \delta+\sin \delta)^{2}} .
$$

Тогда найдется такое $\sigma_{0}>0$, что для любого $-\sigma_{0} \leqslant \sigma<0$ в достаточно малой окрестности неподвижной точки $\left(\xi^{*}, \xi^{*}, \pi\right)^{T}$ имеется орбитально асимптотически неустойчивый цикл, асимптотика которого задается формулой

$$
\begin{aligned}
\left(\xi_{1}(\tau), \xi_{2}(\tau), \alpha(\tau)\right)^{T}= & \sqrt{-\frac{\sigma \phi_{\pi, 0}}{d_{\pi, 0}}} \times \\
& \times\left(\exp \left(\left(i \omega+\sigma\left(\psi_{\pi, 0}-\frac{\phi_{\pi, 0} c_{\pi, 0}}{d_{\pi, 0}}\right)\right) \tau\right)\left(a_{1},-a_{1}, 1\right)^{T}+\kappa . c .\right)+O(\sigma),
\end{aligned}
$$

где

$$
\begin{gathered}
\omega=\frac{1}{2} \sqrt{-1-2 b \operatorname{tg} \delta+\operatorname{tg}^{2} \delta}, \quad a_{1}=-\frac{2 i \omega+\sec ^{2} \delta-2 b \operatorname{tg} \delta}{4 \sqrt{2}(1+i \omega)(2 b-\operatorname{tg} \delta)} \\
\phi_{\pi, 0}+i \psi_{\pi, 0}=2 \cos \delta\left(2-\frac{2 i \omega}{\cos 2 \delta+b \sin 2 \delta}\right) \\
c_{\pi, 0}=-\frac{3 \omega \cos \delta\left(\left(3-13 b^{2}\right) \cos \delta+\left(1+b^{2}\right) \cos 3 \delta+8 b \sin \delta\right)}{8(-2 b \cos \delta+\sin \delta)^{2}(\cos 2 \delta+b \sin 2 \delta)}
\end{gathered}
$$

Приведем кратко сценарий фазовых перестроек, происходящих в системе (13) при $D>0$.

1. При значениях параметра $D>1 /(2 \cos \delta) \approx 0,931(d>\pi(\alpha+1) / 4)$ глобально устойчиво единственное состояние равновесия $\xi_{1}=\xi_{2}=1, \alpha=0$.

2. При $D<1 /(2 \cos \delta)(d<\pi(\alpha+1) / 4)$ к глобально устойчивому состоянию равновесия $(1,1,0)^{T}$ добавляется неустойчивое $\left(\xi^{*}, \xi^{*}, \pi\right)^{T}$.

3. При уменьшении $D$ до значения $D \approx 0,544$ ( $d$ до значения $d \approx 0,459+1,112 \alpha$ ) рождаются еще два устойчивых состояния равновесия - точки $A=\left(\xi_{1}^{*}, \xi_{2}^{*}, \alpha_{1}^{*}\right)^{T}$ и $B=\left(\xi_{2}^{*}, \xi_{1}^{*},-\alpha_{1}^{*}\right)^{T}$, где $\xi_{1}^{*}>\xi_{2}^{*}, 0<\alpha_{1}^{*}<\pi / 2$, и два неустойчивых $-C=\left(\eta_{1}^{*}, \eta_{2}^{*}, \alpha_{2}^{*}\right)^{T}$ и $E=\left(\eta_{2}^{*}, \eta_{1}^{*},-\alpha_{2}^{*}\right)^{T}$, где 
$\eta_{1}^{*}>\eta_{2}^{*}, 0<\alpha_{2}^{*}<\pi / 2$, кроме того, $\eta_{1}^{*}>\xi_{1}^{*}, \xi_{2}^{*}>\eta_{2}^{*}$. Состояния равновесия $A$ и $B$ устойчивы при уменьшении параметра $D$ вплоть до значения $0,524(d \approx 0,442+0,36 \alpha)$, при котором состояния $A$ и $B$ теряют устойчивость с рождением устойчивых циклов $C_{A}$ и $C_{B}$ (бифуркация Андронова-Хопфа).

4. При $D=-\cos \delta-b \sin \delta \approx 0,5015(d=(\pi \alpha-\pi(1-\alpha) \cos \delta(\cos \delta+b \sin \delta)) / 2)$ неустойчивые неподвижные точки $C$ и $E$ сливаются с однородным состоянием равновесия и отбирают его устойчивость.

5. При дальнейшем изменении параметра $D$ устойчивые циклы $C_{A}$ и $C_{B}$, родившиеся из точек $A$ и $B$, увеличиваются в размерах до тех пор, пока при $D \approx 0,481(d \approx 0,219+0,43 \alpha)$ не сомкнутся в точке $\xi_{1}=\xi_{2}=1, \alpha=0$. В результате происходит объединение пары циклов в один $C_{U}$, который остается устойчивым вплоть до значения $D \approx 0,429(d \approx 0,195+0,438 \alpha)$.

6. При $D=1 /(4 \cos \delta) \approx 0,466(d=\pi(1+3 \alpha) / 8)$ от неустойчивого состояния равновесия $\left(\xi^{*}, \xi^{*}, \pi\right)^{T}$ в результате бифуркации Андронова-Хопфа ответвляется неустойчивый цикл $C_{\Pi}$, который при $D \approx 0,429(d \approx 0,195+0,438 \alpha)$ сливается с устойчивым циклом $C_{U}$ и исчезает.

7. При $0<D<0,429(d<0,195+0,438 \alpha)$ система имеет единственное, глобально устойчивое состояние равновесия $\left(\xi^{*}, \xi^{*}, \pi\right)^{T}$.

При $D<0$ получается новая ситуация, не исследованная ранее в статье, которая может быть разрешима при помощи замены (см., например, [5])

$$
\xi_{1} \rightarrow \sqrt{1-2 D \cos \delta} \xi_{1}, \quad \xi_{2} \rightarrow \sqrt{1-2 D \cos \delta} \xi_{2}, \quad \alpha \rightarrow \pi+\alpha .
$$

Замена (21) переводит состояние равновесия $\left(\xi^{*}, \xi^{*}, \pi\right)^{T}$ в $(1,1,0)^{T}$, а $(1,1,0)^{T}$ соответственно в $\left(1 / \xi^{*}, 1 / \xi^{*}, \pi\right)^{T}$. Кроме того, замена времени $s=(1-2 D \cos \delta) s^{*}$ позволит свести систему (13) к идентичной ей с параметром связи

$$
D^{*}=-\frac{D}{1-2 D \cos \delta}
$$

Таким образом, динамика системы (13) при значениях $D<0$ аналогична динамике при $D>0$ с учетом того, что состояния равновесия меняются ролями, а бифуркационные значения параметра $D^{*}$ могут быть вычислены по формуле (22).

Проиллюстрируем полученный аналитический результат численным экспериментом, который выполнен при $\alpha=0,5$. Для этого приведем следующий сценарий фазовых перестроек.

1. При значениях параметра $D<-1,308(0<d<0,234)$ глобально устойчиво состояние равновесия $\xi_{1}=\xi_{2}=1 / \xi^{*}, \alpha=\pi$.

2. При значении $D \approx-1,308(d \approx 0,234)$ рождаются еще два устойчивых состояния равновесия точки $A=\left(\xi_{1}^{*}, \xi_{2}^{*}, \alpha_{1}^{*}\right)^{T}$ и $B=\left(\xi_{2}^{*}, \xi_{1}^{*},-\alpha_{1}^{*}\right)^{T}$, где $\xi_{1}^{*}>\xi_{2}^{*}, 0<\alpha_{1}^{*}<\pi / 2$, и два неустойчивых $C=\left(\eta_{1}^{*}, \eta_{2}^{*}, \alpha_{2}^{*}\right)^{T}$ и $E=\left(\eta_{2}^{*}, \eta_{1}^{*},-\alpha_{2}^{*}\right)^{T}$, где $\eta_{1}^{*}>\eta_{2}^{*}, 0<\alpha_{2}^{*}<\pi / 2$, кроме того, $\eta_{1}^{*}>\xi_{1}^{*}$, $\xi_{2}^{*}>\eta_{2}^{*}$. Состояния равновесия $A$ и $B$ устойчивы при увеличении параметра $D$ вплоть до значения $-1,198(d \approx 0,283)$, при котором состояния $A$ и $B$ теряют устойчивость с рождением устойчивых циклов $C_{A}$ и $C_{B}$ (бифуркация Андронова-Хопфа).

3. При $D=-1,087(d=0,327)$ неустойчивые неподвижные точки $C$ и $E$ сливаются с состоянием равновесия $\left(1 / \xi^{*}, 1 / \xi^{*}, \pi\right)^{T}$ и отбирают его устойчивость.

4. При дальнейшем изменении параметра $D$ устойчивые циклы $C_{A}$ и $C_{B}$, родившиеся из точек $A$ и $B$, увеличиваются в размерах до тех пор, пока при $D \approx-0,995(d \approx 0,365)$ не сомкнутся в точке $\xi_{1}=\xi_{2}=1 / \xi^{*}, \alpha=\pi$. В результате происходит объединение пары циклов в один $C_{U}$, который остается устойчивым вплоть до значения $D \approx-0,796(d \approx 0,449)$.

5. При $D \approx-0,933(d \approx 0,392)$ от неустойчивого состояния равновесия $(1,1,0)^{T}$ в результате бифуркации Андронова-Хопфа ответвляется неустойчивый цикл $C_{\Pi}$, который при $D \approx-0,796$ $(d \approx 0,449)$ сливается с устойчивым циклом $C_{U}$ и исчезает.

6. При $D>-0,796(d>0,449)$ система имеет единственное, глобально устойчивое состояние равновесия $(1,1,0)^{T}$. 
Отметим, что параметры $d$ и $\alpha$ в рамках задачи выбраны положительными, поэтому описанный выше сценарий реализуется полностью лишь при значениях $\alpha^{*}<\alpha<1$, где

$$
\alpha^{*}=-\frac{D_{\pi 1}}{1-D_{\pi 1} \cos \delta} \approx 0,2995, \quad D_{\pi 1} \approx-0,796 .
$$

Перейдем к случаю $\alpha>1$. В этой ситуации в соответствии с леммой 1 нулевое состояние равновесия системы (3) локально асимптотически устойчиво при $d>\pi(1+\alpha) / 4$. Непосредственный численный анализ системы (3) позволяет предположить, что в этом случае нулевое решение глобально асимптотически устойчиво.

Пусть теперь $d<\pi(1+\alpha) / 4$. Нормальная форма (12) в этом случае сводится к виду

$$
\begin{aligned}
\xi_{1}^{\prime} & =-\xi_{1}-\xi_{1}^{3}+D\left(\xi_{2} \cos (\varphi+\delta)-\xi_{1} \cos (\delta)\right), \\
\xi_{2}^{\prime} & =-\xi_{2}-\xi_{2}^{3}+D\left(\xi_{1} \cos (\varphi-\delta)-\xi_{2} \cos (\delta)\right), \\
\varphi^{\prime} & =-b\left(\xi_{2}^{2}-\xi_{1}^{2}\right)-D\left(\frac{\xi_{1}}{\xi_{2}} \sin (\varphi-\delta)+\frac{\xi_{2}}{\xi_{1}} \sin (\varphi+\delta)\right),
\end{aligned}
$$

где

$$
D=\frac{2 d-\pi \alpha}{\pi(\alpha-1)} \sqrt{1+\frac{\pi^{2}}{4}}
$$

Система (23) имеет единственное глобально устойчивое состояние равновесия $\xi_{1}=\xi_{2}=$ $\sqrt{-1-2 D \cos \delta}, \varphi=\pi$. Локальная устойчивость этого состояния равновесия элементарно следует из анализа матрицы устойчивости. А для обоснования глобальной устойчивости требуется провести численный анализ. Этот численный анализ облегчается тем, что для любых начальных условий системы (23) найдется такой момент времени, начиная с которого траектории системы оказываются в цилиндре $\xi_{1}^{2}+\xi_{2}^{2}<2$. Для того чтобы доказать это утверждение, достаточно рассмотреть функцию $\xi_{1}^{2}+\xi_{2}^{2}$ и ее производную в силу первых двух уравнений системы (23).

5. Выводы. В работе изучена динамика системы, состоящей из двух уравнений Хатчинсона с конкурентной и диффузионной связью. Выполнен локальный асимптотический анализ системы в случае близости параметров осцилляторов к значениям, при которых происходит бифуркация Андронова-Хопфа. Проанализирована динамика построенной нормальной формы и приведен полный сценарий фазовых перестроек, происходящих в системе при изменении параметра диффузии. Кроме того, исследована зависимость этого сценария от коэффициента конкурентной связи. В работе найдены значения параметров, при которых в нормальной форме задачи (10) реализуются двухчастотные колебания и колебания в противофазе. Этим режимам соответствуют устойчивые решения системы (1), суммарное среднее по времени которых больше, чем суммарное среднее для однородного цикла. Тем самым при таких значениях параметров соответствующая биологическая система более эффективна.

\section{СПИСОК ЛИТЕРАТУРЫ}

1. Глызин С. Д. Динамические свойства простейших конечноразностных аппроксимаций краевой задачи «реакция-диффузия»// Диффер. уравн. - 1997. - 33, № 6. - С. 805-811.

2. Глызин С. Д. Стационарные режимы одной конечноразностной аппроксимации уравнения Хатчинсона с диффузией// в кн.: Качественные и приближенные методы исследования операторных уравнений. - Ярославль, 1986. - С. 112-127.

3. Глызин С. Д. Учет возрастных групп в уравнении Хатчинсона// Модел. анал. информ. сист. -2007. -14 , № 3. - C. 29-42.

4. Глызин С. Д., Колесов А. Ю. Локальные методы анализа динамических систем. - Ярославль: ЯрГУ, 2006.

5. Горчакова Е. В. Динамика слабого взаимодействия в системе близких видов// Модел. анал. информ. сист. - 2011. - 18, № 1. - С. 68-74.

6. Кащенко C. A. Динамика логистического уравнения с запаздыванием и запаздывающим управлением// Модел. анал. информ. сист. - 2014. - 21, № 5. - С. 61-77. 
7. Колесов Ю. С. Проблема адекватности экологических уравнений/ Деп. в ВИНИТИ 1901-85. - Ярославль: Ярославский государственный университет, 1985.

8. Glyzin S. D. Age groups in Hutchinson equations// Automat. Control Comp. Sci. - 2018. — 52, № 7. P. 714-727.

9. Hutchinson G. E. Circular causal system in ecology// Ann. N.Y. Acad. Sci. - 1948. - 50. - P. 221-246.

Марушкина Елена Александровна

Ярославский государственный университет им. П. Г. Демидова

E-mail: marushkina-ea@yandex.ru

Самсонова Елена Сергеевна

Ярославский государственный университет им. П. Г. Демидова

E-mail: e.s.samsonova@yandex.ru 